\title{
Differences in pressure and temperature transitions of proteins and polymer gels
}

\section{S. Kunugi ${ }^{1}$, K. Kameyama ${ }^{1}$, T. Tada ${ }^{1}$, N. Tanaka ${ }^{1}$, M. Shibayama ${ }^{2}$ and M. Akashi $^{3}$}

\author{
${ }^{1}$ Department of Polymer Science and Engineering, Kyoto Institute of Technology, \\ Matsugasaki, Sakyo, Kyoto, Japan \\ ${ }^{2}$ Institute for Solid State Physics, Neutron Science Laboratory, University of Tokyo, \\ Kashiwa, Chiba, Japan \\ ${ }^{3}$ Department of Molecular Chemistry, Osaka University, Suita, Osaka, Japan
}

\author{
Correspondence \\ S. Kunugi \\ Department of Polymer Science \\ and Engineering, \\ Kyoto Institute of Technology \\ Matsugasaki, Sakyo \\ Kyoto 606-8585 \\ Japan \\ Fax: +81-75-724-7100 \\ E-mail: kunugi@kit.ac.jp \\ Presented at the 3rd International \\ Conference on High Pressure \\ Bioscience and Biotechnology, \\ Rio de Janeiro, RJ, Brazil, \\ September 27-30, 2004
}

Received January 17, 2005 Accepted May 5, 2005

\begin{abstract}
Pressure-driven and temperature-driven transitions of two thermoresponsive polymers, poly(N-isopropylacrylamide) (pNIPAM) and poly(N-vinylisobutyramide) (pNVIBA)), in both a soluble linear polymer form and a cross-linked hydro-gel form, were examined by a dynamic light-scattering method and direct microscopic observation, respectively. Their behavior was compared with that of protein systems. Changes in some characteristic parameters in the time-intensity correlation functions of dynamic light-scattering measurement of aqueous solutions of pNIPAM at various pressures and temperatures showed no essential differences during temperature and pressure scanning and, as a whole, the motions of polymers in aqueous solutions were similar in two types of transitions until chain shrinkage occurred. The gels (cross-linked polymer gels) prepared from the thermoresponsive polymers also showed similar volume transitions responding to the pressure and temperature increase. In temperature transitions, however, gels showed drastic volume shrinkage with loss of transparency, while pressure-induced transition showed a slow recovery of transparency while keeping the size, after first transient drastic volume shrinkage with loss of transparency. At a temperature slightly higher than the transition under atmospheric temperature, socalled reentry of the volume change and recovery of the transparency were observed during the pressure-increasing process, which implies much smaller aggregation or non-aggregated collapsed polymer chains in the gel at higher pressures, indicating a certain mechanistic difference of the dehydration processes induced by temperature and pressure.
\end{abstract}

\section{Introduction}

Pressure-driven protein transition is similar to temperature-driven transition in many aspects and the formulae for the two processes are represented by a simple thermo-
Key words

- Pressure

- Temperature

- Denaturation

- Transition

- Proteins

- Thermoresponsive polymers

- Polymer gel dynamic equation (1-4). We have shown many examples of protein denaturation on a universal P-T plane (5-7).

On the other hand, several studies have shown some fundamental differences between the temperature and pressure transi- 
tions of proteins. These reports have described, in addition to distinct aggregations of proteins through temperature denaturation, a multi-step nature and higher retention of the secondary structures in the pressure transition $(8,9)$.

Several synthetic polymers, including vinyl polymers, are also known to show distinct changes in their molecular level states in solution in response to temperature and pressure, as shown in several examples published by our group (10-18).

In the present study, we examined the temperature and pressure transitions of two thermoresponsive polymers (poly(N-isopropylacrylamide) (pNIPAM) and poly(N-vinylisobutyramide) (pNVIBA)) in both a soluble linear polymer form and a crosslinked hydro-gel form, and compared their behavior with that of proteins.

pNIPAM and pNVIBA are isomers; pNVIBA has amide bonds in its side chains in an inverted direction compared to pNIPAM. Both are also chemical isomers of polyleucine, and thus thermoresponsive polymers and their gels can be considered to be simple but relevant models of proteins. Interestingly, polyleucine barely dissolves in aqueous solutions, while both pNIPAM and pNVIBA are readily soluble in water below the temperature of thermal transition.

Thermodynamically the coil for collapse transition of these polymers upon increasing temperature corresponds to the transition from the unfolded protein to the folded structure, namely a model of the inverse process of cold denaturation across the cold renaturation temperature.

\section{Material and Methods}

The linear polymer pNIPAM was prepared from the $\mathrm{N}$-isopropylacrylamide monomer (Kojin Co., Tokyo, Japan) by radical polymerization and purified by precipitation with ethyl alcohol $\left(\mathrm{M}_{\mathrm{w}}=76,000\right)$.

A cross-linked hydro-gel of N-vinyliso- butyramide was prepared from $\mathrm{N}$-vinylisobutyramide and a cross-linker (butylene-bis$N$-vinylacetamide: 1/100 in monomer ratio) also by radical polymerization.

The cloud points for the aqueous solutions of the polymer were determined by observing the apparent light scattering using a high-pressure optical cell constructed by Teramecs Co. (Kyoto, Japan) with three sapphire windows. The cell was incorporated into a conventional spectrofluorophotometer (RF-5300PC, Shimadzu, Kyoto, Japan), and the excitation and emission light wavelength was $400 \mathrm{~nm}$. The experiments were performed by either scanning the temperature (T) with an electronic regulator linked to a water circulator (Eyela, Tokyo, Japan) at constant pressure, or by scanning the pressure $(\mathrm{P})$ with a compact hand-driven highpressure pump at constant temperature. The starting points were determined by plotting the logarithms of the scattering intensity against $\mathrm{T}$ or $\mathrm{P}$ and fitting to two crossing lines after transforming the intensity values to positive values by adding the necessary minimum integer. The $\mathrm{T}$ or $\mathrm{P}$ values obtained were practically insensitive to the choice of this arbitrary and minimum integer.

The dynamic light scattering (DLS) of aqueous polymer solutions was measured with an ALV-5000 SLS/DLS goniometer (Langen, Germany). A helium-neon laser with a wavelength of $6328 \AA$ was used as an incident beam. The scattered photons were measured with an avalanche photodiode system provided by ALV. A high-pressure sample cell similar to the above mentioned one was settled on the light beam and the scattering was counted at $90^{\circ}$. A $1 \%$ aqueous solution of pNIPAM was prepared and filtered several times through a $0.45-\mathrm{mm}$ MILLEX-HV filter unit (Millipore, Billerica, MA, USA). Pressure and temperature were controlled as in the cloud point measurement.

The time-intensity correlation functions 
$\mathrm{g}^{(2)}(\tau)$ (Equation 1$)$ were analyzed by Equation 2 , consisting of a single exponential function corresponding to the transverse motion of a polymer chain (fast mode) and a stretched exponential function expressing the slow transverse motion of a cluster $(19,20)$.

$\mathrm{g}^{(2)}(\tau)=\left\{\left\langle I(t ; \mathrm{q}) I(t+\tau ; \mathrm{q})>_{\mathrm{T}}\right\} /<I(t ; \mathrm{q})>^{2}{ }_{\mathrm{T}}\right.$

where $I(t ; q)$ is the scattered intensity observed at time $t$ and at the scattering vector q, $\tau$ is the time lag, and \langle\rangle$_{\mathrm{T}}$ denotes the time average.

$\mathrm{g}^{(2)}(\tau)-1 \approx \sigma_{\mathrm{I}}^{2}\left\{A \exp \left(-D_{\mathrm{f}} q^{2} \tau\right)+(1-A) \exp \right.$ $\left.\left[-\left(\tau / \tau_{\mathrm{s}}\right)^{\beta}\right]\right\}^{2}$

(Eq. 2)

where $\sigma_{\mathrm{I}}^{2}, A, \beta, \tau_{\mathrm{s}}$, and $D_{\mathrm{f}}$ are the initial light amplitude, ratio of the slow mode, stretched exponent, relaxation time of the slow mode, and the translational diffusion constant, respectively.

Microscopic observation of the gel under high pressure was performed using a phase difference microscope (OPTHPHOT-2, Nikon, Tokyo, Japan), equipped with a highpressure microscopic cell (PC-400MS, Teramecs). The images were photographed with an USB microscopic camera (QX3, Intel, Santa Clara, CA, USA). Pressure and temperature were controlled as in the cloud point measurement.

\section{Results and Discussion}

In most of the protein denaturation procedures, as shown in a typical example regarding phosphoglycerate kinase (Figure 1), both the pressure transition and the cold transition proceed in a stepwise manner, while these multiple steps occur almost simultaneously in the heat denaturation and the total transition seems to be a single step.

As for a hypothetical double-domain protein, Murphy and Freire (21) described that the free energy states and the structural transitions can be classified into three categories, based on the cooperative free energy between denatured domains $\left(\Delta \mathrm{g}_{\mathrm{A}^{\prime}, \mathrm{B}},\right)$ twostate behavior: 1) both domains are either folded or unfolded, in the case of very large $\left.\Delta \mathrm{g}_{\mathrm{A}^{\prime}, \mathrm{B}^{\prime}}, 2\right)$ independent folding/unfolding of the two domains with very small $\Delta \mathrm{g}_{\mathrm{A}^{\prime}, \mathrm{B}^{\prime}}$, and 3 ) folding/unfolding of interacting domains with intermediate $\Delta \mathrm{g}_{\mathrm{A}^{\prime}, \mathrm{B}}$. Thus, thermal denaturation seems to belong to the first category, but the cold and pressure denaturations belong to the second or the third category.

The time-intensity correlation functions of DLS measurement of aqueous pNIPAM solutions at various pressures and temperatures were obtained as shown in Figure 2. The apparent cloud point of this polymer is known to show an elliptic diagram on the temperature-pressure plane $(10,12)$, and the $\mathrm{T}$ - or P-scanning in the present DLS measurements was performed at $25^{\circ} \mathrm{C}$ or $0.1 \mathrm{MPa}$, respectively, up to the point just before the occurrence of clouding and precipitation.

Changes in some basic parameters $(\beta, A$,

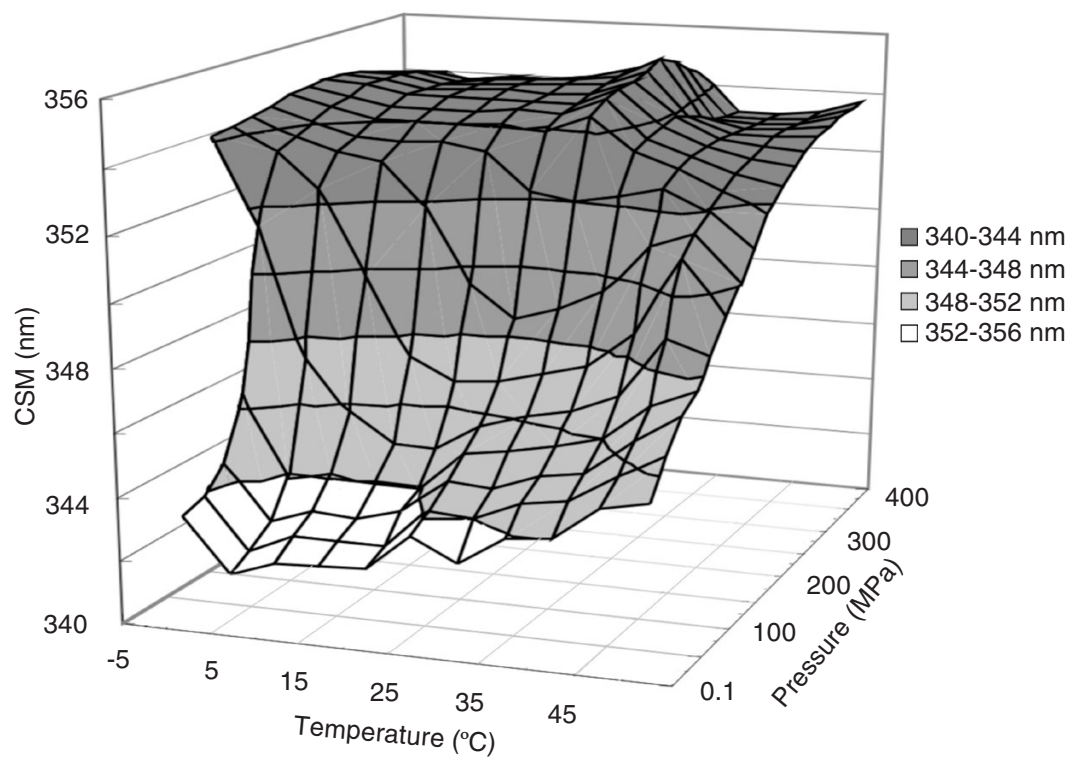

Figure 1. Temperature and pressure dependence of the center of spectrum mass (CSM) of yeast phosphoglycerate kinase fluorescence. $\lambda$ ex $=295 \mathrm{~nm},[\mathrm{E}]=3.5 \mu \mathrm{M}$ in $100 \mathrm{mM}$ Tris$\mathrm{HCl}, \mathrm{pH}=8.0$. 

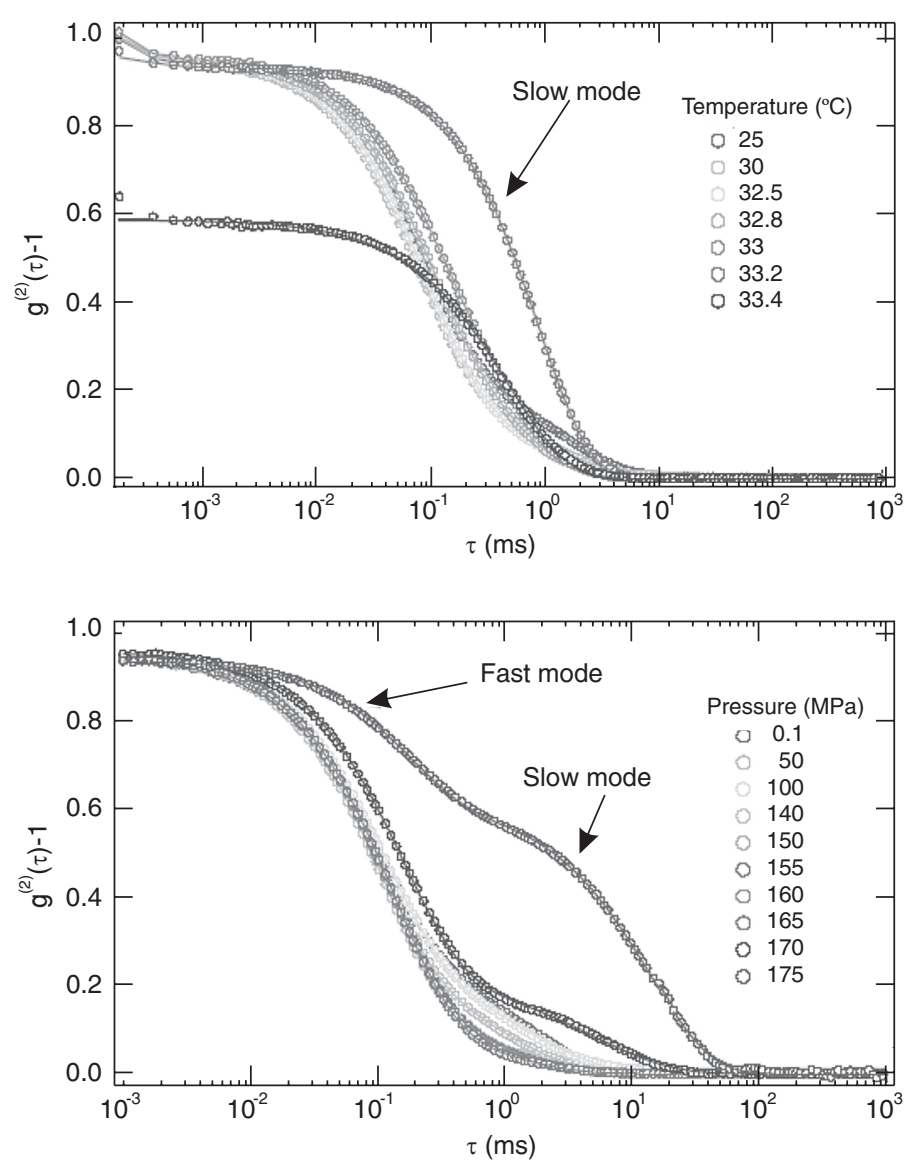

Figure 2. The time-intensity correlation functions of dynamic light scattering measurement of aqueous solutions of poly ( $\mathrm{N}$-isopropylacrylamide) (pNIPAM) at various temperatures and $0.1 \mathrm{MPa}(\mathrm{A})$ and at various pressures and $25^{\circ} \mathrm{C}(\mathrm{B})$. [pNIPAM] $=1 \%$.

Figure 3. Changes in the stretched exponent (B), the ratio of fast mode $(A)$, the diffusion constant $\left(D_{f}\right)$ and the relaxation time of the slow mode $\left(\left\langle\tau_{\mathrm{s}}\right\rangle\right)$ calculated from the data shown in Figure 2, during the temperature change at $0.1 \mathrm{MPa}$ (upper array) and during the pressure changes at $25^{\circ} \mathrm{C}$ (lower array).
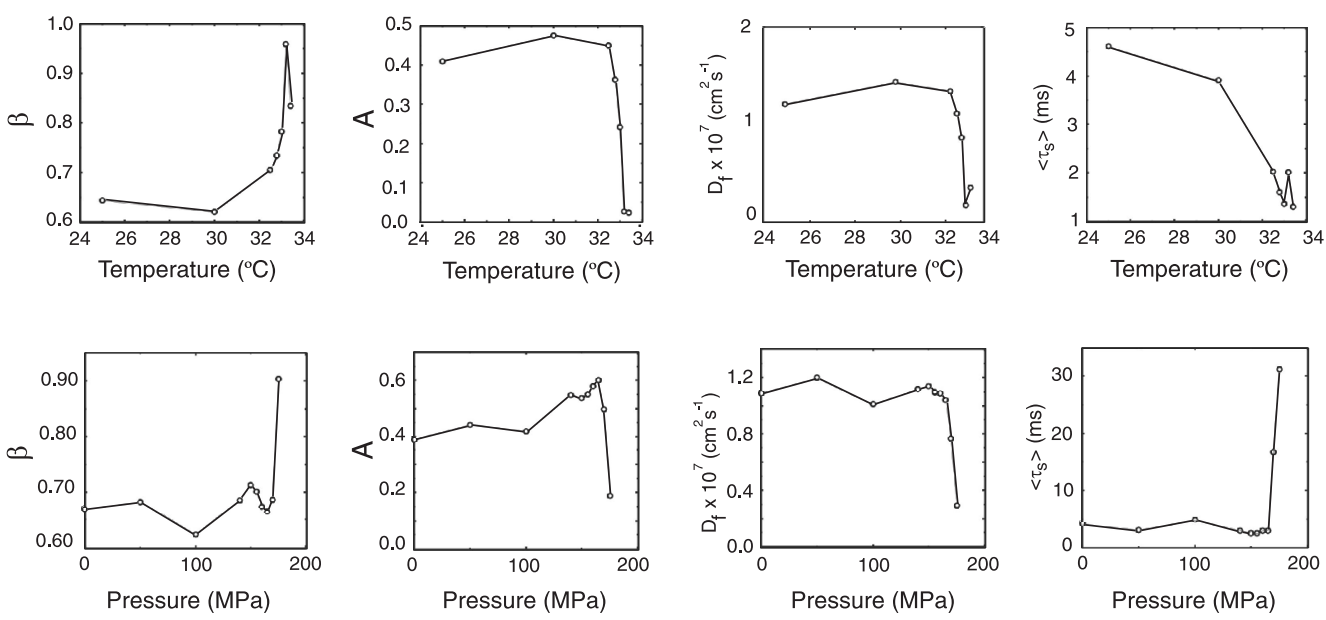

$D_{\mathrm{f}}$, and $\left\langle\tau_{\mathrm{s}}>\right.$ ) were calculated from these time-intensity correlation functions, and those obtained by $\mathrm{T}$ - and $\mathrm{P}$-scanning were compared in Figure 3. No essential differences in the changes in these parameters were observed during temperature and pressure scanning. When approaching the transition point, the stretched exponent $(B)$ increased and the fast mode ratio $(A)$ decreased. The diffusion constant $\left(D_{\mathrm{f}}\right)$ also decreased at higher temperatures or pressures. A difference was observed in the relaxation time of the slow mode $\left.\left(<\tau_{\mathrm{s}}\right\rangle\right)$ which became very low at higher temperatures but higher at higher pressures. On the whole, the motions of polymers in aqueous solutions were similar in the two types of transitions until chain shrinkage occurred.

The gels (cross-linked polymer gels) prepared from the thermoresponsive polymers are known to show a clear volume transition upon exposure to higher temperature (10), reflecting the characteristics of the nature of the polymer component and gel preparation conditions. They also show similar volume transitions responding to the pressure increase. Some examples of microscopic images under various conditions are listed in Figure 4 . With increasing pressure, the transparent gel at $25^{\circ} \mathrm{C}$ gradually shrank and showed a marked volume contraction at pres- 
sures above $400 \mathrm{MPa}$, keeping its transparency. The thermal transition at $0.1 \mathrm{MPa}$ induced a sudden shrinkage between 40 and $41^{\circ} \mathrm{C}$, causing clouding of the gel (the image becomes dark under transmitting white light). The changes at $50 \mathrm{MPa}$ were basically similar to those at $0.1 \mathrm{MPa}$, but the transition occurred at a higher temperature (ca. $45^{\circ} \mathrm{C}$ ). Thus, when we observed at $42^{\circ} \mathrm{C}$ with increasing pressure, the clouded and shrunken gel recovered its size and transparency at, e.g., $25 \mathrm{MPa}$, whereas a further increase in pressure caused the gel to shrink while keeping its transparency. At $49^{\circ} \mathrm{C}$, the clouded and shrunken gel became transparent, keeping its size when the pressure was increased up to $200 \mathrm{MPa}$.

The volumes of the gel at various temperatures and pressures relative to $25^{\circ} \mathrm{C}$ and $0.1 \mathrm{MPa}$ are summarized in the form of contour map in Figure 5. Regarding the volume (or size) of the gel, the figure shows a behavior similar to that of soluble polymers as compared with the transition curves of the soluble pNVIBA illustrated in the same figure. In this respect, $\mathrm{P}$ and $\mathrm{T}$ transitions seem to be the same.

The transitions of these thermoresponsive polymers and gels occur due to the dehydration process and the strengthening of hydrophobic interactions among side chains, and due to the structural deformation of water around the hydrophobic groups.

Hydrophobic interactions among alkyl chains show slightly positive volume changes (22). The compressibility of bulk water is greater than that of the water that surrounds the hydrophobic groups; thus, increasing the

Figure 5. The relative volumes of the poly(N-vinylisobutyramide) (pNVIBA) gel at various temperatures and pressures, presented in the form of a contour map. The reference volume is that at $25^{\circ} \mathrm{C}$ and $0.1 \mathrm{MPa}$. For comparison, the transition curves of the soluble pNVIBA are overlaid: $\mathrm{Mw}=11 \mathrm{kDa}$ (dashed line) and $=66 \mathrm{kDa}$ (dotted line; data from Ref. 12).

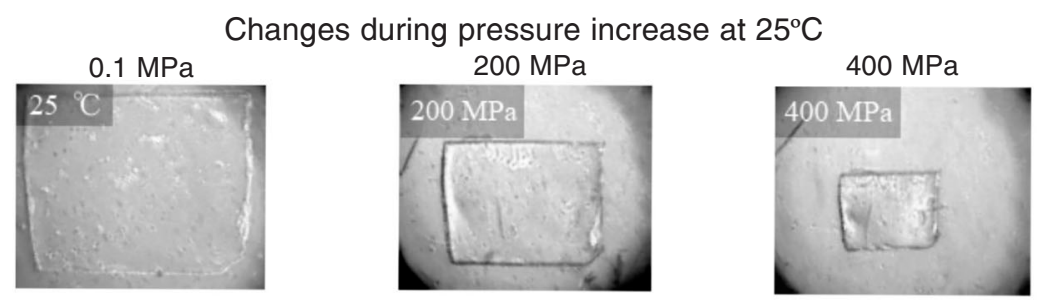

Changes during temperature increase at $0.1 \mathrm{MPa}$
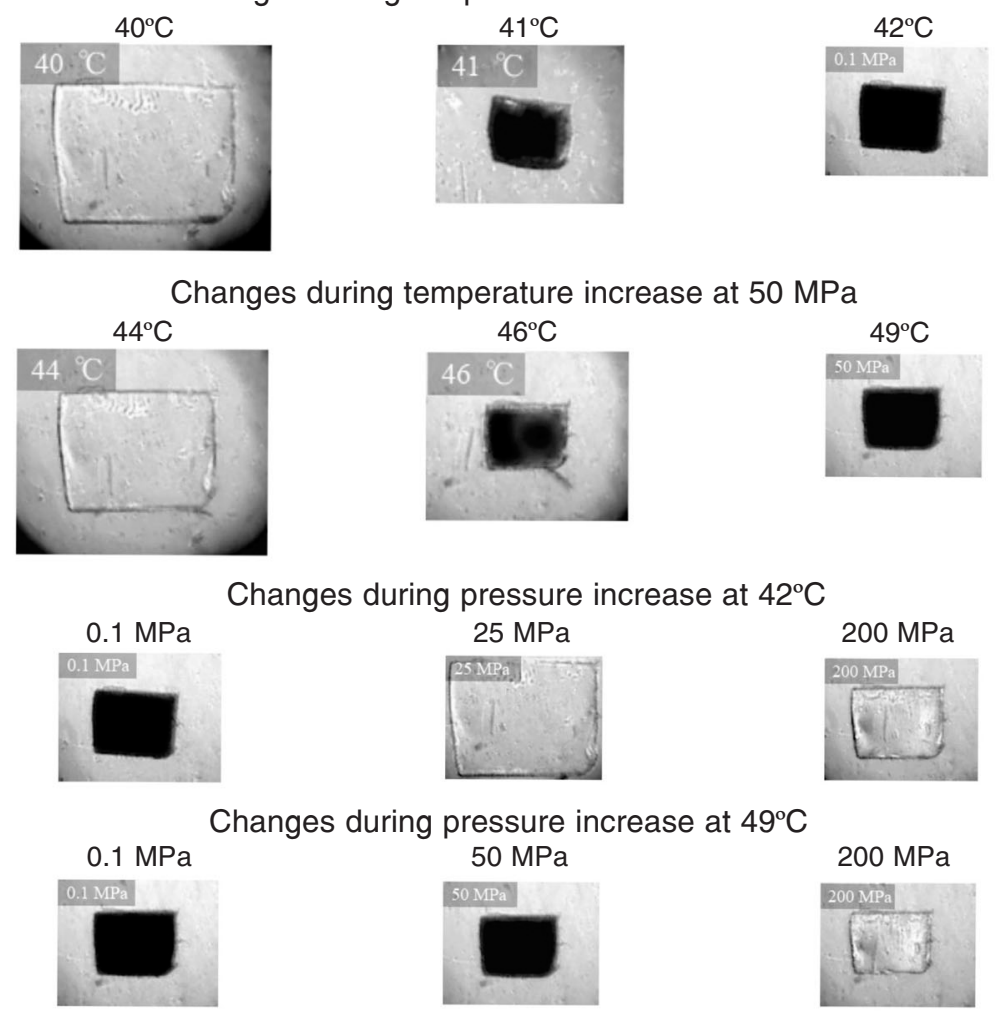

Figure 4. Some examples of microscopic images of the cross-linked poly(N-vinylisobutyramide) ( $\mathrm{pNVIBA}$ ) gels under various conditions.

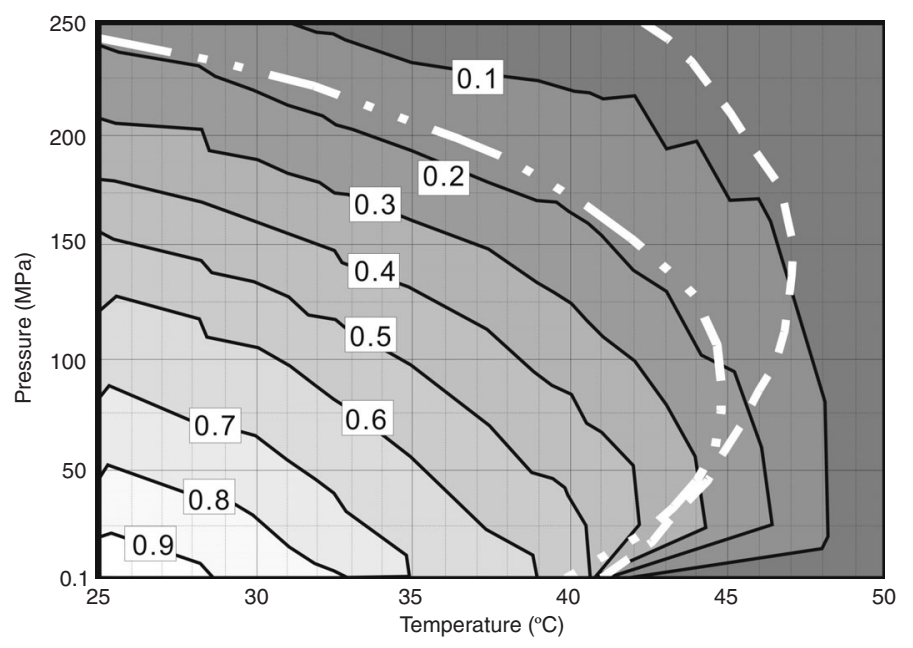


pressure further can invert the situation, and the maximum clouding temperature was observed.

However, the appearance of the shrunken gels at elevated pressures was somehow different from their appearance at lower pressures. At $49^{\circ} \mathrm{C}$, for example, the turbid and shrunken gel became transparent at pressures above $100 \mathrm{MPa}$. This reflects the different aggregation states of the polymer chains. Much less aggregated or non-aggregated collapsed polymer chains will be formed in the gel at higher pressures. This implies a certain mechanistic difference of the dehydration processes induced by temperature and pressure, although we need further precise investigations of the network structures of the shrunken gels in order to understand the detailed mechanisms. Recent studies using molecular dynamic simulation have reported that peptide dehydration differs in the location of the hydrated chains between temperature- or pressure-induced dehydration, which might be a key to detect the mechanism of the present phenomenological differences (23).

\section{References}

1. Hawley SA (1971). Reversible pressure-temperature denaturation of chymotrypsinogen. Biochemistry, 10: 2436-2442.

2. Zipp A \& Kauzmann W (1973). Pressure denaturation of metmyoglobin. Biochemistry, 12: 4217-4228.

3. Heremans K \& Smeller L (1998). Protein structure and dynamics at high pressure. Biochimica et Biophysica Acta, 1368: 353-370.

4. Smeller $L$ (2002). Pressure-temperature phase diagrams of biomolecules. Biochimica et Biophysica Acta, 1595: 11-29.

5. Yamamoto H, Makino M, Tada T et al. (1999). Pressure-assisted cold-denaturation of carboxypeptidase Y. Bulletin of the Chemical Society of Japan, 72: 2803-2806.

6. Ikeuchi H, Kunugi S \& Oda K (2000). Activity and stability of a neutral protease from Vibrio sp. (vimelysin) in a pressure-temperature gradient. European Journal of Biochemistry, 267: 979-983.

7. Tanaka $\mathrm{T}$, Ikeuchi $\mathrm{H}$, Tanaka $\mathrm{N}$ et al. (2004). Pressure-temperatureinduced denaturation of yeast phosphoglycerate kinase, a doubledomain protein. Cellular and Molecular Biology, 50: 317-322.

8. Torrent J, Rubens P, Ribo M et al. (2001). Pressure versus temperature unfolding of ribonuclease A: an FTIR spectroscopic characterisation of 10 variants at the carboxy-terminal site. Protein Science, 10: 725-734.

9. Dzwolak W, Kato M \& Taniguchi $Y$ (2002). Fourier transform infrared spectroscopy in high-pressure studies on proteins. Biochimica et Biophysica Acta, 1595: 131-144.

10. Kunugi S, Takano K, Tanaka $\mathrm{N}$ et al. (1997). Effects of pressure on the behavior of the thermoresponsive polymer poly $(\mathrm{N}$-vinylisobutyramide) (PNVIBA). Macromolecules, 30: 4499-4501.

11. Suwa K, Yamamoto K, Akashi M et al. (1998). Effects of salt on the temperature and pressure responsive properties of poly( $\mathrm{N}$-vinylisobutyramide) aqueous solutions. Colloid and Polymer Science, 276: 529-533.

12. Kunugi S, Yamazaki Y, Takano K et al. (1999). Effects of ionic additives and ionic comonomers on the temperature and pressure responsive behavior of thermoresponsive polymers in aqueous solutions. Langmuir, 15: 4056-4061.

13. Kunugi S, Tada T, Yamazaki $Y$ et al. (2000). Thermodynamic studies on coil-globule transitions of poly $(\mathrm{N}$-vinylisobutyramide-co- vinylamine) (pNVIBA-co-VAm) in aqueous solutions. Langmuir, 16: 2040-2042.

14. Yamazaki Y, Tada T \& Kunugi S (2000). Effects of acrylic acid incorporation on pressure-temperature behavior and calorimetric properties of pNIPAM in aqueous solutions. Colloid and Polymer Science, 278: 80-83.

15. Tamura T, Yamaoka T, Kunugi S et al. (2000). Effects of temperature and pressure on the aggregation properties of an engineered elastin model polypeptide in aqueous solution. Biomacromolecules, 1: 552-555.

16. Kunugi S, Yoshida D \& Kiminami $H$ (2001). Effects of pressure on the behavior of (hydroxypropyl)cellulose in aqueous solution. Colloid and Polymer Science, 279: 1139-1143.

17. Yamaoka T, Tamura T, Seto $Y$ et al. (2003). Mechanism for the phase transition of a genetically engineered elastin model peptide (VPGIG) ${ }_{40}$ in aqueous solution. Biomacromolecules, 4: 1680-1685.

18. Seto $\mathrm{Y}$, Kameyama K, Tanaka $\mathrm{N}$ et al. (2003). High pressure studies on the coacervation of copoly( $\mathrm{N}$-vinylformamide-vinylacetate) and copoly(N-vinylacetylamide-vinylacetate). Colloid and Polymer Science, 281: 690-694.

19. Martin JE, Wilcoxon J \& Odinek J (1991). Decay of density-fluctuations in gels. Physical Review A, 43: 858-872.

20. Okamoto M, Norisuye $T$ \& Shibayama M (2001). Time-resolved dynamic light scattering study on gelation and gel-melting processes of gelatin gels. Macromolecules, 34: 8496-8502.

21. Murphy KP \& Freire E (1992). Thermodynamics of structural stability and cooperative folding behavior in proteins. Advances in Protein Chemistry, 43: 313-361.

22. Suzuki K, Taniguchi Y \& Watanabe $T$ (1973). Effect of pressure on the dimerization of carboxylic acids in aqueous solution. Journal of Physical Chemistry, 77: 1918-1922.

23. Garcia A (2004). Reversible temperature and pressure denaturation of a protein fragment: A replica exchange molecular dynamics simulation study. Abstracts of the 3rd International Conference on High Pressure Bioscience and Biotechnology, Rio de Janeiro, RJ, Brazil, September 26-30, 2004, S18. 